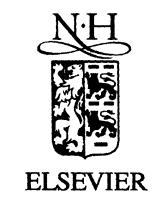

Information Sciences 137 (2001) 189-210

INFORMATION

SCIENCES

AN INIERNATIONAL JOURNAL

www.elsevier.com/locate/ins

\title{
Optimal Gray-code labeling and recognition algorithms for hypercubes
}

\author{
Stavros D. Nikolopoulos \\ Department of Computer Science, University of Ioannina, P.O. Box 1186, GR-45110 Ioannina, \\ Greece
}

Received 2 January 2000; received in revised form 27 November 2000; accepted 18 March 2001

\begin{abstract}
We present an optimal greedy algorithm which returns a Gray-code labeling of the nodes of an $n$-dimensional hypercube; that is, a labeling of the nodes with binary strings of length $n$ for which the Hamming distance between two nodes is 1 if and only if these are adjacent in the hypercube. The proposed algorithm is very simple; it uses breadthfirst search to guide the greedy choice of nodes and computes the Gray-code label of a node $u$ by performing the logical disjunction of the Gray-code labels of two nodes adjacent to node $u$. It takes as input a hypercube $Q_{n}$ with $N=2^{n}$ nodes and runs in $\mathrm{O}(N \log N)$ time. Based on the labeling algorithm we propose a recognition algorithm for hypercubes which runs in $\mathrm{O}(N \log N)$ time. Thus, in view of the fact that $Q_{n}$ has $n 2^{n-1}$ edges, this behaviour is optimal. Both labeling and recognition algorithms incorporate such algorithmic features that they can be optimally implemented in a PRAM model of computation. (C) 2001 Elsevier Science Inc. All rights reserved.
\end{abstract}

Keywords: Hypercube; Gray-code labeling; Recognition; Graph partition; Parallel algorithms; Complexity

\section{Introduction}

With the advances in VLSI technology, it has become feasible to build computing machines with hundreds or even thousands of processors cooperating in solving a given problem. These machines differ along various dimen-

E-mail address: stavros@cs.uoi.gr (S.D. Nikolopoulos). 
sions such as control mechanism, address-space organization, interconnection network, and granularity of processors. Shared memory and non-shared memory (message-passing) parallel machines can be constructed by connecting processors and memory units using a variety of interconnection networks.

Several topologies have been proposed for interconnecting the processors of a non-shared memory parallel computing system. Among them, due to its topological richness, the hypercube topology (also known as $n$-cube, Cosmic cube, Boolean cube) is extremely pervasive in the literature as it provides a structural model for parallel computer architectures; it offers a large bandwidth, logarithmic diameter, and a high degree of fault tolerance $[1,16]$. Both research and commercial systems have been build using the hypercube interconnection scheme, and significant research effort has been devoted to hypercube architectures $[11,13,18]$.

We assume that a hypercube interconnection scheme is represented by its underlying graph, and let $N$ and $n$ be two integers such that $N=2^{n}$. An $n$ dimensional hypercube $Q_{n}=\left(V_{n}, E_{n}\right)$ is an $N$-node graph which is defined recursively as the iterated Cartesian product of the smallest non-trivial complete graph $K_{2}$ consisting of two nodes and one edge joining them:

(i) $Q_{1}=K_{2}$, and

(ii) $Q_{n}=K_{2} \times Q_{n-1}$ for $n \geqslant 2$,

where $\times$ is the Cartesian product of two graphs [7]. Examples of $Q_{1}, Q_{2}, Q_{3}$ and $Q_{4}$ are shown in Fig. 1. It is convenient to say that $Q_{0}=K_{1}$.

Characterizations and topological properties of hypercubes have been extensively studied and interesting results have been reported in the literature $[5,10,16,17]$. In the following, we describe some of the most important
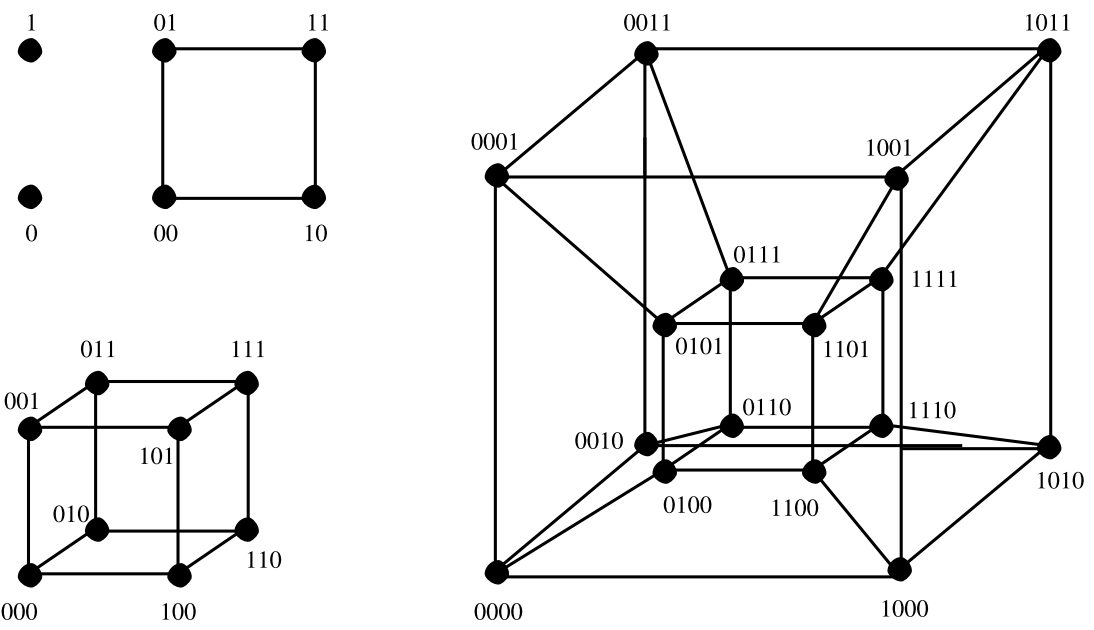

Fig. 1. The first four hypercubes $Q_{1}, Q_{2}, Q_{3}$ and $Q_{4}$. 
characterizations and topological properties of an $n$-dimensional hypercube $Q_{n}$ relating to sparsity, diameter, existence of node disjoint parallel paths, and existence of odd and even cycles.

It is well-known that a characterization of a family $\mathscr{F}$ of graphs gives a necessary and sufficient condition for a given graph $G$ to be in $\mathscr{F}$. For example, $G$ is bipartite if and only if every cycle of $G$ has even length [7]. We next list characterizations for a given graph $G$ to be a hypercube $Q_{n}[5,6,8,12]$. In principle, each of these conditions contains enough information to enable the logical deduction that $G$ is indeed an $n$-cube graph or $n$-dimensional hypercube (we shall only list these criteria and include a reference for each, where the details may be found). The first condition was given by Foldes [5] (see criterion C1). A similar but different condition (see criterion C2) was derived by Garey and Graham [6] in their study of "squashed cubes". Combining their results with the Merger's Theorem [7, p. 47, Theorem 5.9], we easily conclude that the number of node-disjoint $u-v$ paths in a $Q_{n}$ is $d$. The third condition is due to Laborde and Hebbare [12].

(C1) A connected graph $G$ is a hypercube if and only if $G$ is bipartite and the number of geodesics between any two nodes at distance $d$ is $d$ !.

(C2) Graph $G$ is some $Q_{n}$ if and only if $G$ is connected and bipartite and for any two nodes $u, v$ at distance $d$, the connectivity $x(u, v)=d$.

(C3) A connected graph $G$ with $n$ nodes and minimum degree $\delta$ is a hypercube if and only if every pair of adjacent edges lie in a unique 4-cycle and $n=2^{\delta}$.

Let us now focus on the topological properties of a hypercube $Q_{n}$. Some of them can be easily proved or can be immediately derived from characterizations $\mathrm{C} 1-\mathrm{C} 3$. The property $\mathrm{P} 4$ is the most important.

(P1) $Q_{n}$ is a connected bipartite graph.

(P2) $Q_{n}$ has $n 2^{n-1}$ edges.

(P3) The diameter of $Q_{n}$ is $n=\log N$.

(P4) Each node in a $Q_{n}$ can be uniquely represented by an $n$-bit label in such a way that two nodes are adjacent if and only if their labels differ in exactly one bit.

For convenience, we will number the bits in a label of a node of $Q_{n}$ from right to left as 0 to $n-1$. That is, a binary string $b$ of length $n$ will be written as $b_{n-1} b_{n-2} \cdots b_{1} b_{0}$, where $b_{n-1}$ is the most significant bit and $b_{0}$ is the least significant bit. The $i$ th bit (or bit $i$ ) of $b$ is $b_{i}$ for $0 \leqslant i \leqslant n-1$. Fig. 1 shows the hypercubes $Q_{1}, Q_{2}, Q_{3}$ and $Q_{4}$ with a binary labeling of their nodes. If two adjacent nodes differ in their $i$ th bit, then they are said to be in direction $i$ with respect to each other. For example, the node $u$ with label 1011 is said to be in direction 2 of a $Q_{4}$ with respect to node $v$ with label 1111 and vice 
versa. It is clear from this definition that there are $n$ distinct directions in a hypercube $Q_{n}$.

We now consider the labels $s$ and $t$ of two nodes in a $Q_{n}$. The total number of bit positions at which these two labels differ is called the Hamming distance between them [7,11]. For example, the Hamming distance between nodes labeled 011 and 101 in a 3-dimensional hypercube $Q_{3}$ is 2 . Based on the Hamming distance, it is easy to see that the $n$-dimensional hypercubes have the property that two nodes are adjacent if and only if their Hamming distance is 1. Furthermore, taking the property $\mathrm{P} 4$ into account we can define the hypercube $Q_{n}=\left(V_{n}, E_{n}\right)$ as a graph, where $V_{n}$ is the set of all $2^{n}$ binary $n$-strings and $E_{n}$ is precisely those pairs of $n$-strings whose labels vary in exactly one binary digit (see Fig. 2). This presentation is immediately seen to be equivalent (as it must!) to presentation in terms of Cartesian products.

Many research questions that have arisen in the study of binary strings may be stated in terms of hypercube graphs. Perhaps the best known example is that of generating "Gray codes". We define a Gray-code as a sequence of $2^{n}$ binary $n$-strings where successive strings are distinct in exactly one binary digit. Thus, a Gray-code is simply a Hamiltonian cycle on a hypercube and, hence, the number of Gray-codes is the number of Hamiltonian cycles on $Q_{n}$.

A Gray-code labeling of a hypercube is a node labeling for which the Hamming distance between two nodes is 1 if and only if these are adjacent in the hypercube. Gray-code labeling is a fundamental issue of both theoretical and practical importance. Its most important application is related to the portability of algorithms across various parallel architectures. Specifically, with the widespread availability of parallel architectures based on the hypercube
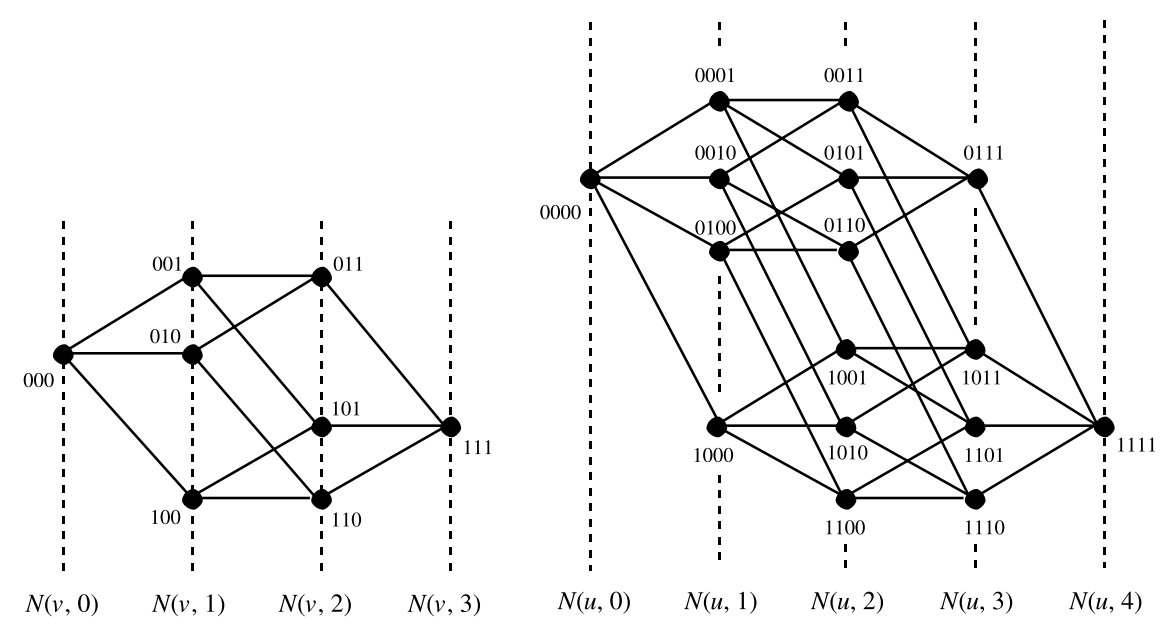

Fig. 2. Partition of the hypercubes $Q_{3}$ and $Q_{4}$, where $v=000$ and $u=0000$. 
interconnection scheme, there is an interest in the portability of algorithms developed for architectures based on other topologies, such as linear arrays, rings, 2-dimensional meshes, and complete binary trees, into the hypercube. This question of portability reduces to one of embedding the above interconnection schemes into the hypercube. Gray-codes (binary reflected Gray-codes) have been extensively used in embedding arrays, rings, meshes and trees into the hypercube.

Our objective is to study the Gray-code labeling and recognition of an $n$-dimensional hypercube and propose optimal sequential and/or parallel algorithms for these problems. Many researchers have extensively studied hypercubes and proposed algorithms for many important problems among which the Gray-code labeling and recognition problems. Recently, Bhagavathi et al. [2] proposed a greedy hypercube-labeling algorithm for Hypercubes on $N=2^{n}$ nodes, which runs in $\mathrm{O}(N \log N)$ time being, therefore, optimal. The main feature of their algorithm is that it uses depth-first search to guide the greedy choice of labels.

In this paper we first present a simple and optimal algorithm for Gray-code labeling of the nodes of an $n$-dimensional hypercube. Specifically, given a hypercube $Q_{n}$ on $N=2^{n}$ nodes, our labeling algorithm visits the nodes of the hypercube by using the breadth-first search and computes the Gray-code label of a node $u$ by performing the logical disjunction of the Gray-code labels of two nodes adjacent to node $u$. It runs in $\mathrm{O}(N \log N)$ time and, therefore, in view of the fact that $Q_{n}$ has $n 2^{n-1}$ edges, this behaviour is optimal. Based on the labeling algorithm we describe an optimal algorithm which recognizes whether a given graph on $N=2^{n}$ nodes is indeed an $n$-dimensional hypercube. Our recognition algorithm runs in $\mathrm{O}(N \log N)$ time being, therefore, optimal.

Moreover, since our algorithms are based on the breadth-first search, they can be efficiently implemented in a PRAM model of computation. More precisely, we present optimal parallel algorithms for the Gray-code labeling and recognition problems. Our main technique is based on the notion of partitioning the vertex set of a hypercube, with respect to a vertex $s$, into a set of (mutually disjoint) adjacency-level sets. We propose two parallel algorithms for Gray-code labeling of a hypercube $Q_{n}$; the first algorithm runs in $\mathrm{O}(\log N)$ time using a total of $\mathrm{O}(N \log N)$ operations on a CRCW PRAM or in $\mathrm{O}\left(\log ^{2} N\right)$ time using a total of $\mathrm{O}(N \log N)$ operations on a CREW PRAM, while the second one is executed on a CREW PRAM in $\mathrm{O}(1)$ time using $\mathrm{O}(N \log N)$ operations, provided that the distance matrix of $Q_{n}$ is given. We also present a parallel algorithm for the recognition problem which runs in $\mathrm{O}(\log N)$ time using $\mathrm{O}(N \log N)$ operations on a CRCW PRAM model or in $\mathrm{O}\left(\log ^{2} N\right)$ time using a total of $\mathrm{O}(N \log N)$ operations on a CREW PRAM model.

The paper is structured as follows. Section 2 presents the main technical results and the key ideas that are at the heart of our optimal hypercube-labeling and recognition algorithms. The labeling and recognition algorithms and their 
complexity analysis are presented in Sections 3 and 4, respectively. Parallel hypercube-labeling and recognition algorithms are described in Section 5. Finally, Section 6 summarizes our results.

\section{The main results}

Given a connected graph $G=(V, E)$ and a vertex $v \in V$, we define a partition $\mathscr{L}(G, v)$ of the vertex set $V$ (we shall frequently use the term partition of the graph $G$ ), with respect to the vertex $v$ as follows:

$$
\mathscr{L}(G, v)=\left\{N_{i}(v)\left|v \in V, 0 \leqslant i \leqslant L_{v}, 0 \leqslant L_{v}<\right| V \mid\right\},
$$

where $N_{i}(v), 0 \leqslant i \leqslant L_{v}$, are the adjacency-level sets, or simply the adjacencylevels, and $L_{v}$ is the length of the partition $\mathscr{L}(G, v)$ [14]. The adjacency-level sets of the partition $\mathscr{L}(G, v)$ of the graph $G$, are formally defined as follows:

$$
N_{i}(v)=\{u \in V|d(v, u)=i, 0 \leqslant i<| V \mid\},
$$

where $d(v, u)$ denotes the distance between vertices $v$ and $u$ in $G$. We point out that $d(v, u) \geqslant 0$, and $d(v, u)=0$ when $v=u$, for every $v, u \in V$. (In the case where $G$ is a disconnected graph, $d(v, u)=\infty$ when $v$ and $u$ do not belong to the same connected component.) Obviously, $L_{v}=\max \{d(v, u) \mid \in V\}$, $N_{0}(v)=\{v\}$ and $N_{1}(v)=N(v)$. In Fig. 2 we illustrate the partitions of the graphs $Q_{3}$ and $Q_{4}$, with respect to the vertices $v$ and $u$, respectively, where $v=000$ and $u=0000$.

Let $G=(V, E)$ be a graph with $n$ nodes and $m$ edges. It is easy to see that, the adjacency-level sets $N_{i}(v), 0 \leqslant i \leqslant L_{v}$, of a partition $\mathscr{L}(G, v)$ can be computed in $\mathrm{O}(n+m)$ time using breadth-first search [4]. Moreover, the adjacencylevel sets of the partition $\mathscr{L}(G, v)$ can easily be computed recursively as follows:

$$
N_{i}(v)=\left\{y \mid(x, y) \in E \text { and } x \in N_{i-1}(v)\right\}-\left\{N_{i-1}(v) \cup N_{i-2}(v)\right\}, \quad 2 \leqslant i \leqslant L_{v} .
$$

(We note that, these sets can also be computed by considering first the distance matrix of the graph $G$ and then extracting all set information that is necessary. This computation can be done by using matrix multiplication; see [3].)

In the rest of the paper we shall denote the adjacency-level sets $N_{0}(v), N_{1}(v), \ldots, N_{i}(v)$ of a partition $\mathscr{L}(G, v)$ as $N(v, 0), N(v, 1), \ldots, N(v, i)$, $0 \leqslant i \leqslant L_{v}$.

Lemma 2.1. Let $Q_{n}=\left(V_{n}, E_{n}\right)$ be an $n$-dimensional hypercube on $N=2^{n}$ nodes, and let $N(v, 0), N(v, 1), \ldots, N(v, n)$ be the adjacency-level sets of the partition $\mathscr{L}\left(Q_{n}, v\right)$, where $v \in V_{n}$. Then, the subgraph induced by the vertex set $N(v, i)$ is a $m K_{1}$ graph, where $m=|N(v, i)|$ and $0 \leqslant i \leqslant n$.

Proof. Immediately from criterion $\mathrm{C} 1$ (or criterion $\mathrm{C} 2$ ). 
Lemma 2.2. Let $Q_{n}=\left(V_{n}, E_{n}\right)$ be an n-dimensional hypercube on $N=2^{n}$ nodes, and let $N(v, 0), N(v, 1), \ldots, N(v, n)$ be the adjacency-level sets of the partition $\mathscr{L}\left(Q_{n}, v\right)$, where $v \in V_{n}$. Let $u, w$ be distinct vertices in $N(v, i), 1 \leqslant i \leqslant n-1$. The following statements must be satisfied:

(a) Nodes $u, w$ have at most one common neighbour in $N(v, i+1)$.

(b) Nodes $u, w$ have a common neighbour in $N(v, i+1)$ if and only if they have a common neighbour in $N(v, i-1)$.

Proof. Both statements follow from criterion C3.

\subsection{Construction properties of a hypercube}

Let $K_{2}$ and $Q_{n}$ be two hypercubes with 2 and $N=2^{n}$ nodes, respectively, $n \geqslant 1$. Let $\left\{v_{1}, v_{2}\right\}$ be the node set of $K_{2}$ and let $\left\{u_{1}, u_{2}, \ldots, u_{N}\right\}$ be the node set of $Q_{n}$. By definition, $Q_{n+1}=K_{2} \times Q_{n}$ is a hypercube with $2 N=2^{n+1}$ nodes. The node set $V\left(Q_{n+1}\right)$ and the edge set $E\left(Q_{n+1}\right)$ of the hypercube $Q_{n+1}$ are the following:

(i) $V\left(Q_{n+1}\right)=\left\{x_{1}, x_{2}, \ldots, x_{N}, y_{1}, y_{2}, \ldots, y_{N}\right\}$, where $x_{i}=\left(v_{1}, u_{i}\right)$ and $y_{i}=$ $\left(v_{2}, u_{i}\right), 1 \leqslant i \leqslant N$

(ii) $\left(x_{k}, y_{k}\right) \in E\left(Q_{n+1}\right)$ for $1 \leqslant i \leqslant N$, and $\left(x_{i}, x_{j}\right),\left(y_{i}, y_{j}\right) \in E\left(Q_{n+1}\right)$ if and only of $\left(u_{i}, u_{j}\right) \in E\left(Q_{n}\right)$ for $1 \leqslant i, j \leqslant N$ and $i \neq j$.

Let $H_{n}$ and $F_{n}$ be two subgraphs of the graph $Q_{n+1}$ induced by $\left\{x_{1}, x_{2}, \ldots, x_{N}\right\}$ and $\left\{y_{1}, y_{2}, \ldots, y_{N}\right\}$, respectively, where $x_{i}=\left(v_{1}, u_{i}\right)$ and $y_{i}=\left(v_{2}, u_{i}\right), 1 \leqslant i \leqslant N$. Let $\mathscr{L}\left(H_{n}, x_{1}\right)$ and $\mathscr{L}\left(F_{n}, y_{1}\right)$ be two partitions of $H_{n}$ and $F_{n}$, respectively, and let

$$
N\left(x_{1}, 0\right), N\left(x_{1}, 1\right), \ldots, N\left(x_{1}, n\right)
$$

and

$$
N\left(y_{1}, 0\right), N\left(y_{1}, 1\right), \ldots, N\left(y_{1}, n\right)
$$

be the adjacency-level sets of these partitions. Moreover, let $\mathscr{L}\left(Q_{n+1}, s\right)$ be a partition of $Q_{n+1}$ with respect to node $s=x_{1}$, and let $N(s, 0)$, $N(s, 1), \ldots, N(s, n+1)$ be the adjacency-level sets of $\mathscr{L}\left(Q_{n+1}, s\right)$. Then, the following hold:

(i) $N(s, 0)=N\left(x_{1}, 0\right), N(s, n+1)=N\left(y_{1}, n\right)$, and $N(s, i)=N\left(x_{1}, i\right) \cup N\left(y_{1}, i-1\right)$, $1 \leqslant i \leqslant n$.

(ii) There is one and only one edge $\left(x_{k}, y_{k}\right) \in E\left(Q_{n+1}\right)$ such that $x_{k} \in V\left(H_{n}\right)$ and $y_{k} \in V\left(F_{n}\right), 1 \leqslant k \leqslant N$ (see criterion C3). Moreover, if node $x_{k} \in N(s, i)$ then node $y_{k} \in N(s, i+1)$, where $0 \leqslant i \leqslant n$.

Next, we describe a Gray-code labeling method which, as we shall see later, will be used as a basis for the proposed Gray-code labeling algorithm. For simplicity, hereafter, we shall say that a hypercube is Gray-code labeled or $G$-labeled if the $n$-bit labels of its nodes satisfy the property P4. Moreover, we shall refer to the Gray-code label of a node $u$ as $G$-label $(u)$. 


\subsection{Gray-code labeling of a hypercube}

Let $K_{2}$ and $Q_{n}$ be two hypercubes and let $\left\{v_{1}, v_{2}\right\}$ and $\left\{u_{1}, u_{2}, \ldots, u_{N}\right\}$ be the node sets of $K_{2}$ and $Q_{n}$, respectively, where $N=2^{n}$ and $n \geqslant 1$. We assume that both hypercubes $K_{2}$ and $Q_{n}$ are $G$-labeled. That is, if $G$-label $(u)$ denotes the Gray-code label of the node $u$, then we have:

(i) $G$-label $\left(v_{1}\right)=0$,

(ii) $G$-label $\left(v_{2}\right)=1$, and

(iii) $G$-label $\left(u_{i}\right)=b_{n-1} b_{n-2} \cdots b_{1} b_{0}$, where $b_{n-1} b_{n-2} \cdots b_{1} b_{0}$ be an $n$-bit string such that the Hamming distance between two nodes is 1 if and only if the nodes are adjacent in the hypercube $Q_{n}$.

Let $X=b_{n-1} b_{n-2} \cdots b_{1} b_{0}$ be a binary string of length $n$, where $b_{n-1}$ is the most significant bit and $b_{0}$ is the least significant bit. Then, $0(X)$ (resp. $1(X)$ ) denotes the binary string $b_{n} b_{n-1} b_{n-2} \cdots b_{1} b_{0}$ of length $n+1$, where $b_{n}=0$ (resp. $\left.b_{n}=1\right)$.

We have seen that $Q_{n+1}=K_{2} \times Q_{n}$ and $V\left(Q_{n+1}\right)=\left\{x_{1}, x_{2}, \ldots, x_{N}, y_{1}\right.$, $\left.y_{2}, \ldots, y_{N}\right\}$, where $x_{i}=\left(v_{1}, u_{i}\right)$ and $y_{i}=\left(v_{2}, u_{i}\right), 1 \leqslant i \leqslant N$. We label the nodes of the hypercube $Q_{n+1}$ as follows:

(i) $\operatorname{label}\left(x_{i}\right)=0(X)$

(ii) label $\left(y_{i}\right)=1(X)$, where $X=G$-label $\left(u_{i}\right), 1 \leqslant i \leqslant N$.

It is easy to see that the assignment of labels which is performed by the above hypercube-labeling method forms a Gray-code labeling of the hypercube $Q_{n+1}$. We call this hypercube-labeling method GL-method and a hypercube labeled by the $G L$-method GL-labeled hypercube.

Let $V\left(Q_{n+1}\right)=\left\{u_{1}, u_{2}, \ldots, u_{2 N}\right\}$ be the node set of the hypercube $Q_{n+1}$ and let $H_{n}$ and $F_{n}$ be two subgraphs of $Q_{n+1}$ induced be the sets $V\left(H_{n}\right)$ and $V\left(F_{n}\right)$, where $V\left(H_{n}\right)$ (resp. $V\left(F_{n}\right)$ ) contains all the nodes $u$ of $Q_{n+1}$ whose the most significant bit of $G$-label $(u)$ is 0 (resp. 1). It is easy to see that $H_{n}$ and $F_{n}$ are two $n$-dimensional hypercubes on $N=2^{n}$ nodes. It is also easy to see that if we assign a new label to each node $u$ of the hypercubes $H_{n}$ and $F_{n}$ by deleting the most significant bit of the $G$-label $(u)$, then both hypercubes $H_{n}$ and $F_{n}$ are $G$-labeled. Hereafter, the hypercubes $H_{n}$ and $F_{n}$ will be referred to as upper hypercube and lower hypercube of the hypercube $Q_{n+1}$, respectively.

The main properties of the construction and $G$-labeling of a hypercube are summarized in the following lemma.

Lemma 2.3. Let $Q_{n+1}$ be a GL-labeled hypercube with $N=2^{n+1}$ nodes and let $G$ label $(u)$ be the Gray-code label of the node $u$, where $u \in V\left(Q_{n+1}\right)$. We partition the hypercube $Q_{n+1}$ with respect to node $s$, where $G$-label $(s)=00 \cdots 0$, and let $N(s, 0), N(s, 1), \ldots, N(s, n+1)$ be the adjacency-level sets of $Q_{n+1}$. Then, the following hold:

(i) $V\left(Q_{n+1}\right)=V\left(H_{n}\right) \cup V\left(F_{n}\right)$, where $H_{n}$ and $F_{n}$ are the upper and lower n-dimensional hypercubes of $Q_{n+1}$. 
(ii) The most significant bit of the G-label(u) of a node $u \in V\left(Q_{n+1}\right)$ is 0 (resp. 1) if and only if $u \in V\left(H_{n}\right)$ (resp. $u \in V\left(F_{n}\right)$ ).

(iii) Every node $v$ of the set $V\left(H_{n}\right)$ is joined by an edge with one and only one node $u$ of the set $V\left(F_{n}\right)$ (see criterion C3). Moreover, if $G$-label $(v)=0(X)$ then $G$-label $(u)=1(X)$, where $X$ is a binary string of length $n$.

Next, we present the main theorem of this paper which provides the justification for an algorithm which assigns $G$-labels to a hypercube $Q_{n}$.

Theorem 2.1. Let $Q_{n}=\left(V_{n}, E_{n}\right)$ be an n-dimensional hypercube on $N=2^{n}$ nodes, and let $N(s, 0), N(s, 1), \ldots, N(s, n)$ be the adjacency-level sets of the partition $\mathscr{L}\left(Q_{n}, s\right)$, where $s \in V_{n}$. Let $u, v$ be distinct nodes in $N(s, i)$ and let $w$ be a node in $N(s, i+1)$ such that $(w, u) \in E_{n}$ and $(w, v) \in E_{n}, 1 \leqslant i \leqslant n-1$. Then, G-label(w) $=G$-label $(u) \vee G$-label(v) is a Gray-code label of the node $w$.

Proof. We shall prove the theorem by induction on the dimension $n$ of the hypercube $Q_{n}$. Let $K_{2}$ and $Q_{1}$ be two hypercubes and let $V\left(K_{2}\right)=\{s, u\}$ and $V\left(Q_{1}\right)=\{v, w\}$ be their node sets. Let $Q_{2}=K_{2} \times Q_{1}$. Thus, $V\left(Q_{2}\right)=\{s, u, v, w\}$ and let $\{s, u\}$ and $\{v, w\}$ be the node sets of the upper and lower hypercubes of $Q_{2}$, respectively. We assign the label 00 to the node $s$, that is, $G$-label $(s)=00$. Then, $G$-label $(u)=01$ and $G$-label $(v)=10$. Obviously, $G$-label $(w)=$ $G$-label $(u) \vee G$-label $(v)=11$ and $w \in N(s, 2)$. Therefore, in the base case $n=2$ the induction hypothesis holds.

Assume that the induction hypothesis holds for a hypercube $Q_{n-1}$ on $N=2^{n-1}$ nodes, $n \geqslant 2$. We shall show that it also holds for $Q_{n}$. Let $Q_{n}=\left(V_{n}, E_{n}\right)$ be an $n$-dimensional hypercube on $N=2^{n}$ nodes, and let $N(s, 0), N(s, 1), \ldots, N(s, n)$ be the adjacency-level sets of the partition $\mathscr{L}\left(Q_{n}, s\right)$, where $s \in V_{n}$. Moreover, let $H_{n}$ and $F_{n}$ be the upper and lower hypercubes of $Q_{n}$. Then, $H_{n}$ and $F_{n}$ are two hypercubes each on $N=2^{n-1}$ nodes.

Suppose that we have labeled the nodes of the levels $N(s, 0)$, $N(s, 1), \ldots, N(s, i)$ of the partition $\mathscr{L}\left(Q_{n}, s\right)$, by using the given condition. Let $w$ be a node in $N(s, i+1)$ and let $w \in V\left(F_{n}\right)$. Let $w^{\prime}$ be a node such that $w^{\prime} \in V\left(F_{n}\right), w^{\prime} \in N(s, i),\left(w^{\prime}, w\right) \in E_{n}$ and $G$-label $\left(w^{\prime}\right)=0(X \vee Y)$; see Fig. 3. We shall prove that $G$-label $(w)=1(X \vee Y)$.

It is easy to see that all the labeled neighbours of the node $w^{\prime}$ belong to the upper hypercube. Thus, by the induction hypothesis we have $0(X \vee Y)=\cdots=$ $0(X \vee Z)=\cdots=0(Y \vee Z)$. We distinguish four cases:

Case I: label $(w)=\operatorname{label}(u)$ OR label $(v)=(1 \vee 1)(X \vee Y)=1(X \vee Y)$.

Case II: label $(w)=\operatorname{label}(u)$ OR $\operatorname{label}(q)=(1 \vee 1)(X \vee Z)=1(X \vee Y)$.

Case III: $\operatorname{label}(w)=\operatorname{label}(u)$ OR $\operatorname{label}\left(w^{\prime}\right)=(1 \vee 0)(X \vee(X \vee Y))=$ $1(X \vee Y)$. 


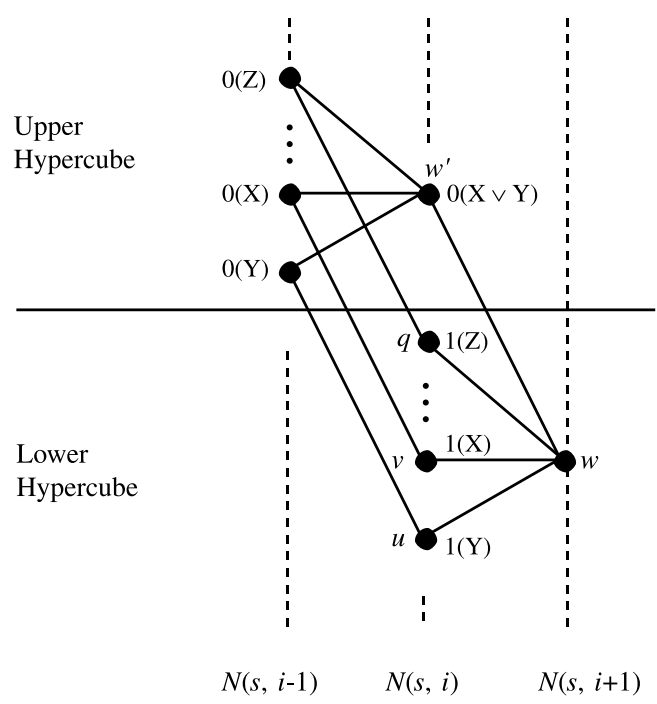

Fig. 3. Three consecutive adjacency-levels of the partition $\mathscr{L}\left(Q_{n}, s\right)$.

\section{Case IV:}

$$
\begin{aligned}
\operatorname{label}(w)=\operatorname{label}(q) \text { OR label }\left(w^{\prime}\right) & =(1 \vee 0)(Z \vee(X \vee Y))=1((Z \vee X) \vee Y) \\
& =1((X \vee Y) \vee Y)=1(X \vee Y) .
\end{aligned}
$$

So indeed label $(w)$ is a $G$-label of the node $w$ of the hypercube $Q_{n}$. Thus, the theorem follows for all values of $n$.

\section{The Gray-code labeling algorithm}

We now present an optimal algorithm for Gray-code labeling of the nodes of an $n$-dimensional hypercube. The basic idea of the algorithm is motivated by the characterizations provided by Theorem 2.1. The algorithm is very simple and uses breadth-first search and logical disjunction on $n$-bit strings; it visits the nodes of the hypercube by using the breadth-first search and computes the $G$-label of a node $u$ by performing the logical disjunction of the $G$-labels of two nodes adjacent to node $u$. More precisely, the algorithm takes as input the adjacency list of an $n$ dimensional hypercube $Q_{n}=\left(V_{n}, E_{n}\right)$ with $N=2^{n}$ nodes and operates as follows:

(1) Select an arbitrary node $s \in V_{n}$ and label node $s$ and its adjacent nodes properly; obviously, this process computes the $G$-labels of all the nodes of the sets $N(s, 0)$ and $N(s, 1)$.

(2) Determine the next non-G-labeled node $u$ of the set $N(s, i)$ by using breadth-first search, $2 \leqslant i \leqslant \log N$. 
(3) Select two arbitrary nodes $a$ and $b$ of the set $N(s, i-1)$ which are adjacent to $u$, and compute the $G$-label of $u$ by performing the logical disjunction of $G$-label $(a)$ and $G$-label $(b)$.

From the above described Gray-code labeling method, it is clear that the $G$ label of a node $u$ is the binary representation of $G$-label $(a) \vee G$-label $(b)$, where $\checkmark$ is the logical OR operation and $a, b$ are two Gray-code labeled nodes which are adjacent to node $u$.

The nodes of the hypercube $Q_{n}$ are visited in the order in which they are selected in stage (2) of the algorithm. This visiting process fixes the $G$-labels of the nodes of $Q_{n}$. According to the process of visiting the nodes of $Q_{n}$, the labeling of the nodes of the adjacency-level set $N(s, i)$ cannot start, unless all nodes of set $N(s, i-1)$ are labeled. Thus, the two nodes $a$ and $b$, which are arbitrarily selected of the set $N(s, i-1)$ in stage (3), are always $G$-labeled. The correctness of the algorithm is established through the Theorem 2.1. Its proof relies on the results of Section 2 (see Lemmas 2.1-2.3).

Having described the Gray-code labeling algorithm and proved its correctness, we are now in a position to estimate its time complexity. In doing so we give a more formal listing of the algorithm (see Fig. 4).

Let us first comment on some important features of the algorithm. For each node $u$ of $Q_{n}$ we maintain four variables: $G$-label $(u), \operatorname{Color}(u), \mathrm{A}(u)$ and $\mathrm{B}(u)$. The variable $\operatorname{Color}(u)$ takes the colors White, Gray or Black, and the variables $A(u)$ and $B(u)$ maintain the predecessors of the node $u$ from the previous adjacency levels. To keep track of progress, first the algorithm colors each node White (unprocessed), then Gray (in queue) and finally Black (processed); actually, the coloring process is due to breadth-first search.

Initially, Color $(u)$ is White for every node $u$ of $Q_{n}$; it becomes Black, after the computation of the $G$-label of a node $w$. Consequently, the algorithm determines the node $u$ which is adjacent to $w$ and has White or Gray color. Then, it assigns the node $w$ to $A(u)$, if $A(u)=u$ (if a node $u^{\prime} \neq u$ has been assigned to $A(u)$, then the algorithm assigns the node $w$ to $B(u)$, again if $B(u)=u)$. If $u$ is a White node, then $u$ is inserted into the queue $\mathrm{Q}$ (enqueue operation) and is colored Gray. Later, the algorithm determines the Gray node $u$ at the head of the queue $\mathrm{Q}$, computes the $G$-label $(u)$ of the node $u$ by performing the logical disjunction of $G$-label $(A(u))$ and $G$-label $(B(u))$, deletes $u$ from the queue Q (dequeue operation) and colors it Black. Since, the algorithm inserts only White nodes into the queue Q and then colors them Gray, we easily conclude that each node of the hypercube is enqueued at most once, and hence dequeued at most once. For simplicity, the $n$-bit string consisting of all zeros will be denoted by $0^{n}$.

We shall assume a generic one-processor Random Access Machine (RAM) model of computation as our implementation technology. In this model, the operations of enqueuing and dequeuing take $\mathrm{O}(1)$ time, while both logical $O R$ 
Algorithm G-labeling;

begin

1. For each $u \in V_{\mathrm{n}}$ do $\operatorname{Color}(u) \leftarrow$ White; $\mathrm{A}(u) \leftarrow \mathrm{B}(u) \leftarrow u$;

2. $\quad$ Choose an arbitrary node $s \in V_{\mathrm{n}}$; $G$-label $(s) \leftarrow 0^{\text {n }}$; Color $(s) \leftarrow$ Black;

3. $G$-label $\left(u_{\mathrm{i}}\right) \leftarrow 0^{\mathrm{n}}$, for every node $u_{\mathrm{i}} \in \operatorname{adj}(s), 0 \leq i \leq n-1$;

4. For each node $u_{\mathrm{i}} \in \operatorname{adj}(s), 0 \leq i \leq n-1$, do

4.1 update $G$-label $\left(u_{\mathrm{i}}\right)$ by toggling its $i$-th bit, and then assign Color $\left(u_{\mathrm{i}}\right) \leftarrow$ Black;

4.2 for each node $v \in \operatorname{adj}\left(u_{\mathrm{i}}\right)$ do

$$
\begin{aligned}
& \text { if } \mathrm{A}(v)=v \text { then } \mathrm{A}(v) \leftarrow u_{\mathrm{i}} \\
& \text { elseif } \mathrm{B}(v)=v \text { then } \mathrm{B}(v) \leftarrow u_{\mathrm{i}} ; \\
& \text { if } \operatorname{Color}(v)=\text { White then Enqueue }(\mathrm{Q}, v) \text {; } \operatorname{Color}(v) \leftarrow \text { Gray; }
\end{aligned}
$$

5. While $\mathrm{Q} \neq \varnothing$ do

$5.1 \quad u \leftarrow \operatorname{head}(\mathrm{Q})$;

$5.2 G$-label $(u) \leftarrow G$-label $(\mathrm{A}(u))$ OR $G$-label $(\mathrm{B}(u))$;

5.3 for each node $v \in \operatorname{adj}(u)$ do

if $\operatorname{Color}(v) \neq$ Black then

$$
\text { if } \begin{aligned}
& \mathrm{A}(v)=v \text { then } \mathrm{A}(v) \leftarrow u \\
& \text { elseif } \mathrm{B}(v)=v \text { then } \mathrm{B}(v) \leftarrow u ;
\end{aligned}
$$

if $\operatorname{Color}(v)=$ White then Enqueue $(\mathrm{Q}, v)$; $\operatorname{Color}(v) \leftarrow$ Gray;

end;

\subsection{Dequeue $(\mathrm{Q})$; Color $(u) \leftarrow$ Black;}

Fig. 4. The Gray-code labeling algorithm for $n$-dimensional hypercubes.

operation $x \vee y$ and assignment operation $x \leftarrow y$ take $\mathrm{O}(\log N)$ time in the case where $x$ and $y$ are binary $n$-strings.

Let us now analyze the computational complexity of the algorithm. We shall obtain its overall complexity by computing the complexity of each step separately. The complexity of the algorithm is analyzed as follows: Step 1. Clearly, this initialization step takes $\mathrm{O}(N)$ time, where $N=2^{n}$. Step 2. Since $G$-label is a binary $n$-string, the main operation of this step requires $\mathrm{O}(\log N)$ time. Step 3. Based on the operation of step 2, it follows that this step requires $\mathrm{O}\left(\log ^{2} N\right)$ time. Step 4. The for loop of this step is executed $k$ times, $0 \leqslant k \leqslant n-1$. Thus, steps 4.1 and 4.2 are executed exactly $\log N$ times. It is easy to see that step 4.1 requires $\mathrm{O}(\log N)$ time. The for loop of step 4.2 is executed in $\mathrm{O}(\log N)$ time. In total, step 4 is executed in $\mathrm{O}\left(\log ^{2} N\right)$ time. Step 5. The while loop of this step iterates as long as there remain White nodes in the hypercube. Since each node of the hypercube is enqueued at most once, and hence dequeued at most once, steps 5.1-5.4 are executed exactly $N$ times. It is easy to verify that steps 5.1 and 5.4 take $\mathrm{O}(1)$ time, while steps 5.2 and 5.3 take $\mathrm{O}(\log N)$ time. Thus, step 5 is executed in $\mathrm{O}(N \log N)$ time. 
Taking into consideration the time complexity of each step of the algorithm, we conclude that the total running time of the algorithm is bounded by $\mathrm{O}(N \log N)$. In view of the fact that a hypercube $Q_{\mathrm{n}}$ has $n 2^{n-1}$ edges, this behaviour is optimal. The results of this section can be summarized in the following theorem.

Theorem 3.1. Given an n-dimensional hypercube $Q_{n}$ on $N=2^{n}$ nodes, the algorithm G-labeling correctly produces a Gray-code labeling of $Q_{n}$ in $O(N \log N)$ time.

\section{The recognition algorithm}

In this section, we present an optimal algorithm for the problem of recognizing $n$-dimensional hypercubes. Our hypercube labeling algorithm G-labeling is used as a basis for the proposed recognition algorithm.

Let $\mathscr{L}\left(Q_{n}, s\right)$ be a partition of an $n$-dimensional hypercube $Q_{n}=\left(V_{n}, E_{n}\right)$, with respect to node $s \in V_{n}$, and let $N(s, 0), N(s, 1), \ldots, N(s, n)$ be the adjacency-levels of $\mathscr{L}\left(Q_{n}, s\right)$. The following properties hold.

(P5) The adjacency-levels $N(s, 0), N(s, 1), \ldots, N(s, n)$ are independent sets (see Lemma 2.1).

(P6) The adjacency-level set $N(s, p)$ has $n ! /(p !(n-p) !)$ nodes, $0 \leqslant p \leqslant n$.

(P7) Each node of the set $N(s, p)$ has $p$ adjacent nodes in $N(s, p-1)$ and $n-p$ adjacent nodes in $N(s, p+1), 1 \leqslant p \leqslant n-1$.

Next, we show the relationship between the $G$-label of a node of the level $N(s, p)$ and its distance of the nodes of the level $N(s, 1), 2 \leqslant p \leqslant n$. We prove the following result.

Lemma 4.1. Let $Q_{n}=\left(V_{n}, E_{n}\right)$ be an $n$-dimensional hypercube on $N=2^{n}$ nodes, and let $N(s, 0), N(s, 1), \ldots, N(s, n)$ be the adjacency-level sets of the partition $\mathscr{L}\left(Q_{n}, s\right)$, where $s \in V_{n}$. Let $u$ and $v_{p}$ be nodes of the sets $N(s, 1)$ and $N(s, p)$, respectively, $2 \leqslant p \leqslant n$, and let $G$-label $(u)=0 \cdots 1 \cdots 0$, where 1 holds the ith position, $0 \leqslant i \leqslant n-1$. Then, the G-label $\left(v_{p}\right)$ has 1 in the ith position if and only if $d\left(u, v_{p}\right)=p-1$.

Proof. It follows directly from Theorem 2.1, since all the nodes of a path $\left[u, v_{2}, \ldots, v_{p}\right]$ have 1 in the $i$ th position of their $G$-labels, where $u \in N(s, 1)$ and $v_{j} \in N(s, j), 2 \leqslant j \leqslant p$ (see Fig. 2 or Fig. 5(a)).

Given an $n$-dimensional hypercube $Q_{n}$ and the adjacency-level sets $N(s, 0), N(s, 1), \ldots, N(s, n)$ of the partition $\mathscr{L}\left(Q_{n}, s\right)$, we can easily show the following properties: 


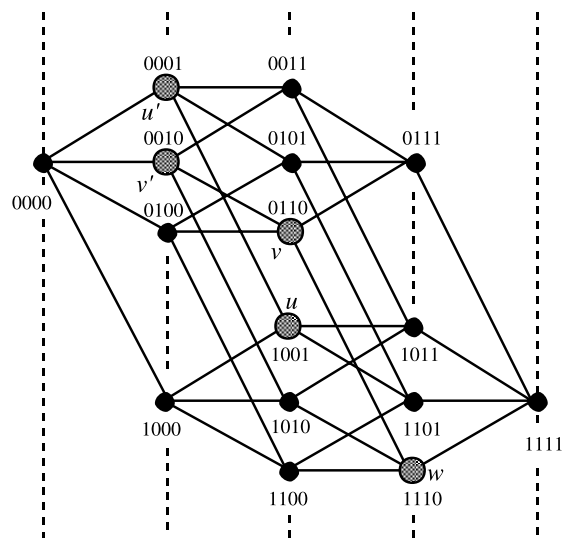

(a)

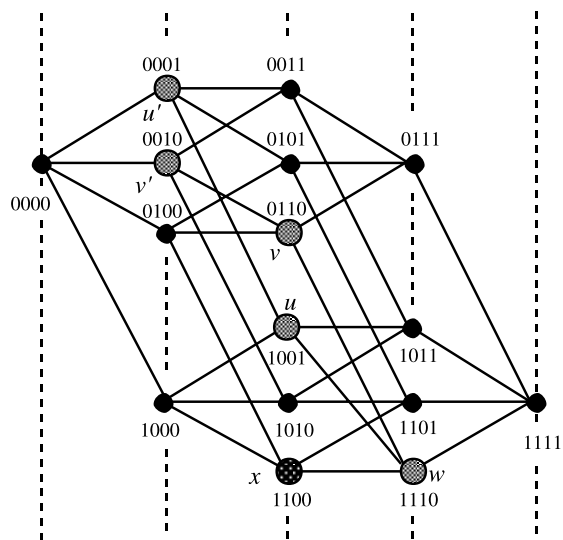

(b)

Fig. 5. (a) The hypercube $Q_{4}$; (b) A graph $Q$ on $N=2^{4}$ nodes; $Q$ is not a hypercube.

(P8) For every node $v$ in the set $N(s, p), 2 \leqslant p \leqslant n$, there exist exactly $p$ nodes $u$ in the set $N(s, 1)$ such that $d(u, v)=p-1$, (see criterion $\mathrm{C} 2$ and property $\mathrm{P} 6$ ).

(P9) The $G$-label of every node $v$ of the set $N(s, p)$ has exactly $p 1$ 's and, hence, $n-p$ 0's (see Lemma 4.1 and Theorem 2.1).

(P10) The $G$-labels of every pair of nodes $u, v$ of the set $N(s, p)$ differ in exactly two bits if $u, v$ have a common neighbour in the set $N(v, p-1), 1 \leqslant p \leqslant n-1$; otherwise, their $G$-labels differ in at least four bits, $n \geqslant 4$ (see Lemma 2.1 and Theorem 2.1).

We are now in a position to prove the following theorem which gives a necessary and sufficient condition for a given graph on $N=2^{n}$ nodes to be an $n$ dimensional hypercube. Hereafter, we shall refer to the integer representation of the $G$-label of a node $u$ as $I G$-label $(u)$.

Theorem 4.1. Let $Q=(V, E)$ be a graph on $N=2^{n}$ nodes which satisfies the properties P5-P7, and let IG-label $\left(v_{i}\right)$ be the integer representation of the G-label of the node $v_{i} \in V, 0 \leqslant i \leqslant N-1$. Then, $Q$ is an $n$-dimensional hypercube if and only if for every $w \in V$ the adjacent nodes $u_{0}, u_{1}, \ldots, u_{n-1}$ of $w$ can be ordered such that $\mid I G$-label $(w)-I G$-label $\left(u_{k}\right) \mid=2^{k}, 0 \leqslant k \leqslant n-1$.

Proof. $(\Rightarrow)$ Since $Q$ is a hypercube on $N=2^{n}$ nodes, each node $w$ in $Q$ can be uniquely represented by an $n$-bit string using the algorithm $\mathrm{G}$-labeling; that is, $G$-label $(w)$. Thus, this implication follows directly from the properties P4 and P9 (see Fig. 5(a)). 
$(\Leftarrow)$ Suppose now that $Q$ is not a hypercube, and let $N(s, 0), N(s, 1), \ldots, N(s, n)$ be the adjacency-level sets of the partition $\mathscr{L}(Q, s)$, where $s \in V$. Since $Q$ satisfies the properties P5-P7, it follows that there exists a pair of adjacent edges which do not form a 4-cycle in $Q$. Therefore, there exists a node $w$ in $N(s, p)$ and two neighbours $u, v$ of $w$ in $N(s, p-1)$ such that the pair of adjacent edges $(u, w)$ and $(w, v)$ do not form a 4-cycle in $Q$; that is, nodes $u, v$ do not have a common neighbour in $N(s, p-2)$ (see Fig. 5(b); $p=3$ ). Suppose that $N(s, p)$ is the first adjacency-level set which contains such a node $w$. There are two cases to consider:

Case I: $G$-label $(w)=G$-label $(v) \vee G$-label $(x)$, where nodes $v, x$ have a common neighbour in $N(s, p-2)$; clearly, $x \neq u$. The $G$-labels of the nodes $v, x$ differ in exactly two bits (see property P10) and, thus, $G$-label(w) and $G$-label $(v)$ differ in exactly one bit. Since $u, v$ do not have a common neighbour in $N(s, p-2)$, it follows that $G$-label $(u)$ and $G$-label $(v)$ differ in at least four bits. Thus, $G$-label $(v)$ and $G$-label(w) differ in at least two bits.

Case II: $G$-label $(w)=G$-label $(u) \vee G$-label $(v)$. Since $G$-label $(u)$ and $G$-la$\operatorname{bel}(v)$ differ in at least four bits, it follows that $G$-label $(u)$ and $G$-label(w) differ in at least two bits.

In both cases, we conclude that there exists a neighbour $u$ of $w$ such that $G$-label $(u)$ and $G$-label $(w)$ differ in more than one bit and, thus, $\mid I G$-label $(w)-I G-\operatorname{label}(u) \mid \neq 2^{k}, 0 \leqslant k \leqslant n-1$.

Based on the conditions provided by Theorem 4.1, we next develop a recognition algorithm for Hypercubes. Obviously, these conditions contain enough information to enable the logical deduction that a given graph $Q=(V, E)$ on $N=2^{n}$ nodes is indeed an $n$-dimensional hypercube. We call the recognition algorithm G-recognition and give its formal listing in Fig. 6.

Let us now analyze the computational complexity of the recognition algorithm. We shall follow a step-by-step analysis. Step 1 . We have proved that the time complexity of the algorithm $\mathrm{G}$-labeling is $\mathrm{O}(N \log N)$; see Theorem 3.1. Moreover, we have seen that this algorithm computes the adjacency-level sets of the input graph. Within the recognition algorithm, we can easily modify the labeling algorithm $\mathrm{G}-\mathrm{labeling}$ so that it computes the adjacency-levels of any graph $Q$ in $\mathrm{O}(N \log N)$ time; the algorithm terminates in the case where $Q$ is not an $n$-dimensional hypercube. Step 2 . Since an $n$-dimensional hypercube has $N$ nodes and $N \log N$ edges, it is easy to see that for any graph $Q$ we can decide whether the properties P5-P7 hold in $\mathrm{O}(N \log N)$ time. Step 3. Given the $G$-labels of the nodes of $Q$, we can easily compute the corresponding integer labels of $Q$ in $\mathrm{O}(N \log N)$ time. Step 4. Obviously, it takes $\mathrm{O}(N)$ time. Step 5. The for loop of this step is executed for every node $w \in V$. Each of the for loops of steps $5.1-5.3$ is executed exactly $\log N$ times. Thus, the total computational time of step 5 is $\mathrm{O}(N \log N)$. Step 6 . This step returns the message " $Q$ is a hypercube"; hence, it takes $O(1)$ time. 


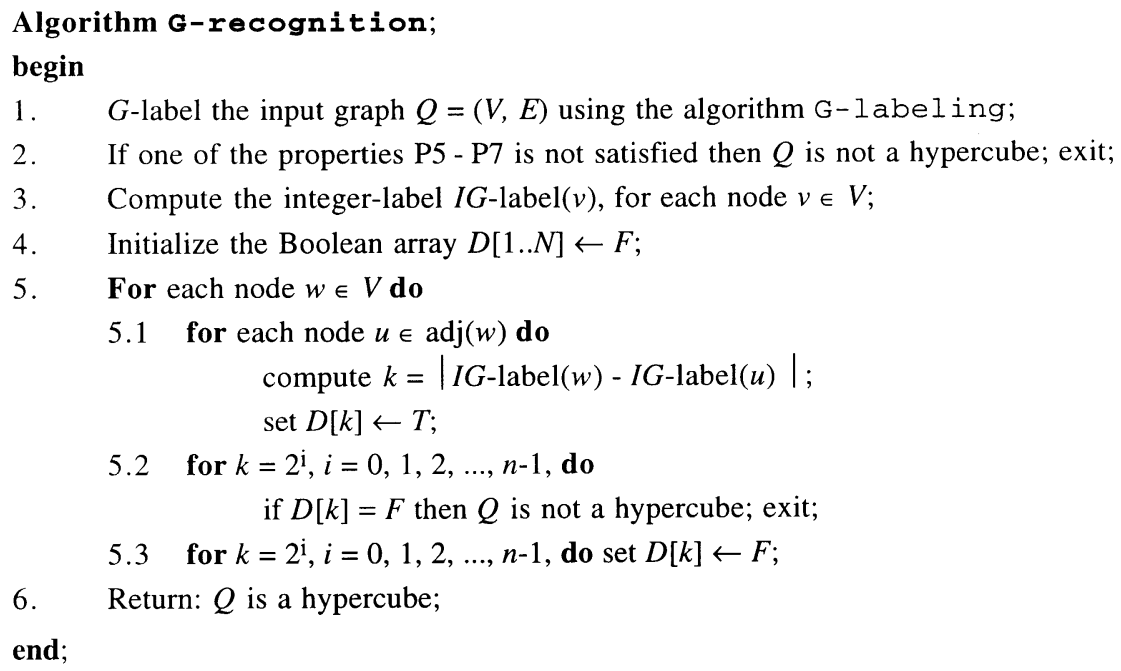

Fig. 6. The recognition algorithm for $n$-dimensional hypercubes.

Taking into consideration the time complexity of each step of the algorithm, we conclude that the algorithm runs in $\mathrm{O}(N \log N)$ time being, therefore, optimal. Recall that a hypercube $Q_{n}$ has $n 2^{n-1}$ edges. Thus, we have proved the following result.

Theorem 4.2. Given a graph $Q$ with $N=2^{n}$ nodes and $n 2^{n-1}$ edges, the algorithm $\mathrm{G}$-recognition recognizes whether $Q$ is an $n$-dimensional hypercube in $\mathrm{O}(N \log N)$ time.

\section{Parallel implementation}

In this section, we present optimal parallel Gray-code labeling and recognition algorithms for $n$-dimensional hypercubes. We present two labeling algorithms; the first algorithm is a parallel implementation of the sequential algorithm G-labeling described in Section 3, while the basic idea of the second one is motivated by the characterizations provided by Lemma 4.1.

For the design and analysis purposes we shall use the Parallel Random Access Machine (PRAM) as a model of parallel computation [9,15]. Our notion of optimality is based on the Work-Time (WT) framework [9]. The work is defined to be the total number of operations used by a parallel algorithm (it has nothing to do with the number of processors available), while the time is defined to be the total number of time units required by a parallel algorithm (during each time unit a number of concurrent operations can take place). 
Within the WT framework, a parallel algorithm for solving a given computational problem will be called optimal if the work $W(n)$ required by the algorithm is asymptotically the same as the sequential complexity of the problem, regardless of the running time $T(n)$ of the parallel algorithm.

\subsection{Parallel labeling algorithms}

We next describe the parallel algorithm $P G-1$ abeling-A which is a parallel implementation of the optimal sequential algorithm G-labeling in Fig. 7.

The correctness of the algorithm PG-labeling-A is established through the correctness of the sequential algorithm $\mathrm{G}$-labeling. Next, we analyze the computational complexity of the algorithm on a CRCW PRAM model.

We follow a step-by-step analysis.

Step 1. Clearly, this initialization step can be executed in $\mathrm{O}(1)$ time using $\mathrm{O}(N)$ operations, where $N=2^{n}$.

Step 2. This step can be completed in $\mathrm{O}(1)$ time using $\mathrm{O}(\log N)$ operations, since the length of the binary $n$-string $G$-label is $n=\log N$.

Step 3. The for loop is executed concurrently for each node $u$ in the adjacency set $\operatorname{adj}(s)$; the set $\operatorname{adj}(s)$ contains $\log N$ nodes. It is easy to see that, both steps 3.1 and 3.2 are executed in $\mathrm{O}(1)$ time using $\mathrm{O}(\log N)$ and $\mathrm{O}(1)$ operations, respectively. The for loop of step 3.3 is executed in $\mathrm{O}(1)$ parallel time using $\mathrm{O}(\log N)$ operations. Thus, overall, step 3 is executed in $\mathrm{O}(1)$ time using $\mathrm{O}\left(\log ^{2} N\right)$ operations.

Step 4. The for loop of this step is executed $\mathrm{O}(\log N)$ times. In its $i$ th iteration, the number of the Gray nodes selected in step 4.1 are exactly

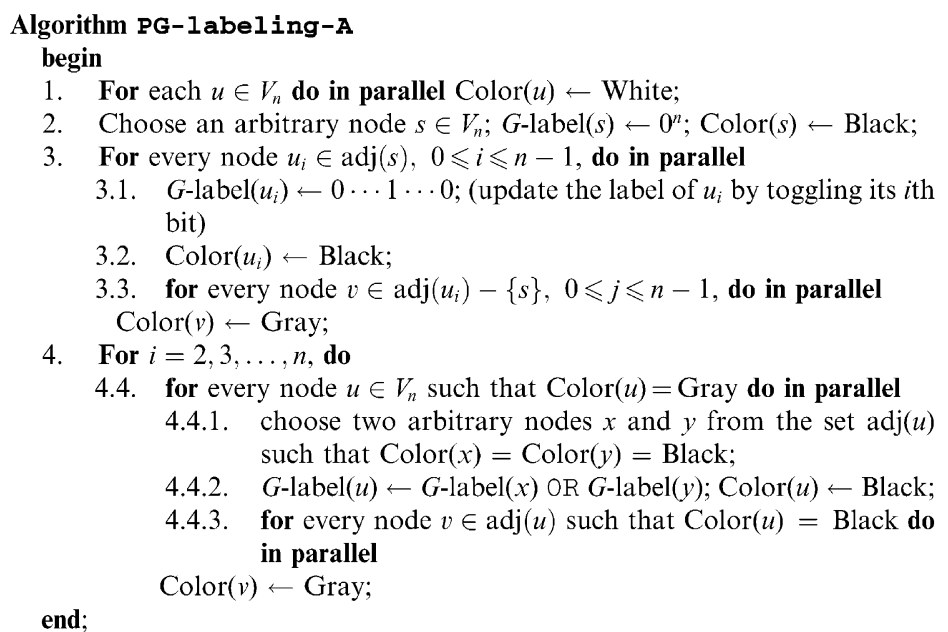

Fig. 7. The parallel Gray-code algorithm PG-labeling-A. 
$k_{i}=|N(s, i)|, i=2,3, \ldots, n$. It is easy to verify that, all the statements of step 4.1 can be executed in $\mathrm{O}(1)$ time using $\mathrm{O}(\log N)$ operations. Thus, step 4 is executed in $\mathrm{O}(\log N)$ time using $k_{2} \log N+k_{3} \log N+\cdots+k_{n} \log N$ operations. Since $k_{2}+k_{3}+\cdots+k_{n}=N-(\log N+1)$, the total number of operations used by step 4 is $\mathrm{O}(N \log N)$.

The time complexity of each step of the algorithm, as well as the number of operations used by each step, imply the following result.

Theorem 5.1. Given an n-dimensional hypercube $Q_{n}$ on $N=2^{n}$ nodes, the parallel algorithm $\mathrm{PG}$-labeling-A correctly produces a Gray-code labeling of $Q_{n}$ in $\mathrm{O}(\log N)$ time using a total of $\mathrm{O}(N \log N)$ operations on a $C R C W$ PRAM.

Algorithm PG-labeling-A requires a concurrent write of the same value capability to run in $\mathrm{O}(\log N)$ time; see, statement $\operatorname{Color}(v) \leftarrow$ Gray in steps 3.3 and 4.1.3. This follows from the fact that each node $v$ is adjacent to more that one node with black color. Since our analysis is based on this assumption, our $\mathrm{O}(\log N)$ time parallel algorithm requires the common $\mathrm{CRCW}$ PRAM model. Our algorithm is optimal because its total number of operations is asymptotically the same as the complexity of the optimal sequential labeling algorithm.

However, it is easy to see that simultaneous memory access to an entry Color( $v$ ) for the purpose of writing can be avoided by executing steps 3.3 and 4.1.3 sequentially. This modification yields an $\mathrm{O}\left(\log ^{2} N\right)$ time parallel algorithm which can be executed on the CREW PRAM model using a total of $\mathrm{O}(N \log N)$ operations. Thus, we also have a parallel labeling algorithm which is optimal for the CREW PRAM model.

Moreover, we can easily modify Algorithm PG-labeling-A so that it computes the adjacency-level sets of the partition $\mathscr{L}\left(Q_{n}, v\right)$; this can be done by adding in step 4.1.1 the statement level $(u) \leftarrow \operatorname{level}(x)+1$, or level $(u) \leftarrow \operatorname{level}(y)+1$. Thus, we have the following results:

Theorem 5.2. A Gray-code labeling of an n-dimensional hypercube $Q_{n}$ on $N=2^{n}$ nodes can be optimally produced in $\mathrm{O}\left(\log ^{2} N\right)$ time using a total of $\mathrm{O}(N \log N)$ operations on the CREW PRAM.

Corollary 5.1. Let $Q_{n}$ be an n-dimensional hypercube on $N=2^{n}$ nodes and let $v$ be an arbitrary node. The adjacency-level sets $N(v, 0), N(v, 1), \ldots, N(v, n)$ can be computed in $\mathrm{O}(\log N)$ time using $\mathrm{O}(N \log N)$ operations on a $C R C W P R A M$ or in $\mathrm{O}\left(\log ^{2} N\right)$ time using $\mathrm{O}(N \log N)$ operations on the CREW PRAM.

Next we describe a parallel labeling algorithm which is mainly based on the relationship between the $G$-label of a node of level $N(s, p)$ and its distance from the nodes of level $N(s, 1)$. Recall that the $G$-label of a node $v$ of the set $N(s, p)$ 


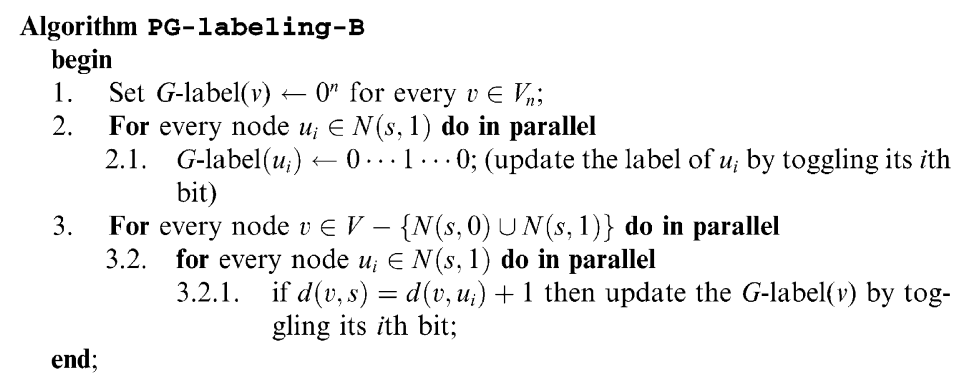

Fig. 8. The parallel Gray-code algorithm PG-labeling-B.

has 1 in the $i$ th position if and only if there exists a node $u$ in the set $N(s, 1)$ such that $d(u, v)=p-1$ and $G$-label $(u)$ has 1 in the $i$ th position, $2 \leqslant p \leqslant n$; see Theorem 4.1. Thus, if the distance matrix of hypercube $Q_{n}$ is given, we can compute a Gray-code labeling of $Q_{n}$ using the following algorithm given in Fig. 8.

The complexity of the $P G-l a b e l i n g-B$ algorithm is analyzed in a step-bystep fashion as follows:

Step 1. Obviously, this step takes $\mathrm{O}(1)$ time using $\mathrm{O}(N \log N)$ operations.

Step 2. The adjacency-level set $N(s, 1)$ contains $\log N$ nodes and, thus, this step is executed in $\mathrm{O}(1)$ time with $\mathrm{O}(\log N)$ operations.

Step 3. The number of nodes in the sets $V-\{N(s, 0) \cup N(s, 1)\}$ and $N(s, 1)$ are $N-(\log N+1)$ and $\log N$, respectively. The operation of testing whether the $i$ th bit of the $G$-label of a node needs to be updated can be sequentially executed in $\mathrm{O}(1)$ time. Therefore, step 3 is completed in $\mathrm{O}(1)$ time using $\mathrm{O}(N \log N)$ operations.

Thus, from the above analysis, we obtain the overall computational complexity of the algorithm; it runs in $\mathrm{O}(1)$ time and uses a total of $\mathrm{O}(N \log N)$ operations.

It is easy to see that steps 1 and 2 of the algorithm can be executed on the EREW PRAM model. However, step 3 requires a concurrent read of the same value $u_{i} \in N(s, 1)$ and, thus, the whole algorithm requires the CREW PRAM model. Thus, the following theorem holds.

Theorem 5.3. Given an n-dimensional hypercube $Q_{n}$ on $N=2^{n}$ nodes and its distance matrix, a Gray-code labeling of $Q_{n}$ can be produced in $\mathrm{O}(1)$ time using a total of $\mathrm{O}(N \log N)$ operations on a CREW PRAM model.

Remark 5.1. It is well-known that the $N \times N$ distance matrix of a graph can be computed using matrix multiplication (matrix powers) [9]. Thus, the matrix $D$ of a hypercube $Q_{n}$ can be computed in $\mathrm{O}(\log N \log \log N)$ time using $\mathrm{O}(M(N) \log \log N)$ operations on the CREW PRAM model, where $M(N)$ is the best known sequential bound for multiplying two $N \times N$ matrices. 


\subsection{Parallel recognition of hypercubes}

In this section we show that the problem of recognizing whether a given graph $Q$ on $N=2^{n}$ nodes is an $n$-dimensional hypercube can be optimally solved in parallel. We present an optimal parallel recognition algorithm which is based on the conditions given by Theorem 4.1; it is a parallel implementation of the sequential algorithm G-recognition described in Section 4.

Obviously, the step 1 of the algorithm G-recognition can be implemented using the parallel algorithm PG-labeling-A. Moreover, we can easily show that steps 3 through 6 of the same algorithm can be implemented in $\mathrm{O}(\log N)$ time using a total of $\mathrm{O}(N \log N)$ operations on a CREW PRAM model.

Let us now focus on the parallel implementation of step 2 of the sequential algorithm G-recognition. Given the adjacency-levels $N(s, 0)$, $N(s, 1), \ldots, N(s, n)$ of the partition $\mathscr{L}(Q, s)$, we can decided whether each of these sets is an independent set in $\mathrm{O}(1)$ time using a total of $\mathrm{O}(N \log N)$ operations on a CRCW PRAM model. It is easy to see that, we can count the elements of the sets $N(s, 0), N(s, 1), \ldots, N(s, n)$ in $\mathrm{O}(\log N)$ time using $\mathrm{O}(N)$ operations on the EREW PRAM model. Moreover, we can test whether a node $u$ of the set $N(s, p)$ has $p$ adjacent nodes in the set $N(s, p-1)$ and $n-p$ adjacent nodes in the set $N(s, p+1)$ in $\mathrm{O}(\log N)$ time using $\mathrm{O}(N \log N)$ operations on a CREW PRAM model, $1 \leqslant p \leqslant n-1$.

Taking into consideration the above analysis, as well as the computational complexity of the parallel labeling algorithms, we state the following theorem.

Theorem 5.4. Let $Q=(V, E)$ be a graph on $N=2^{n}$ nodes. The problem of recognizing whether $Q$ is an n-dimensional hypercube can be solved in $\mathrm{O}(\log N)$ time using a total of $\mathrm{O}(N \log N)$ operations on a CRCW PRAM model or in $\mathrm{O}\left(\log ^{2} N\right)$ time using a total of $\mathrm{O}(N \log N)$ operations on a CREW PRAM model.

\section{Conclusions}

In this paper we presented an optimal greedy algorithm for Gray-code labeling and recognizing $n$-dimensional hypercubes. Our labeling algorithm uses breadth-first search to guide the greedy choice of nodes and computes the Gray-code label of a node $u$ by performing the logical disjunction of the Graycode labels of two nodes adjacent to node $u$. The algorithm is very simple and runs in $\mathrm{O}(N \log N)$ time, where $N=2^{n}$ is the number of nodes in the hypercube.

The idea of our algorithm is motivated by the work performed by Bhagavathi et al. [2]. They presented an optimal algorithm which takes as input an 
$n$-dimensional hypercube $Q_{n}$ and returns a Gray-code labeling of the nodes of the hypercube $Q_{n}$. The main feature of their algorithm is that it visits the nodes of the hypercube by using depth-first search. Based on the labeling algorithm we proposed an optimal recognition algorithm for hypercubes; that is, our algorithm recognizes whether a given graph on $N=2^{n}$ nodes is indeed an $n$-dimensional hypercube and runs in $\mathrm{O}(N \log N)$ time.

It is well-known that the depth-first search is a hardly parallelisable problem; it is a P-complete problem. On the other hand, it is also well-known that the breadth-first search can be efficiently implemented in parallel using many techniques, such as matrix multiplication, vertex collapse, etc $[9,15]$.

Based on these facts, as well as on the properties of the $G L$-method, we proposed an $\mathrm{O}(\log N)$ time CRCW PRAM and an $\mathrm{O}\left(\log ^{2} N\right)$ time CREW PRAM optimal parallel algorithms for the Gray-code labeling problem. Moreover, we proposed another labeling parallel algorithm; it takes advantage of certain topological properties of a hypercube $Q_{n}$ and runs in $\mathrm{O}(1)$ time using $\mathrm{O}(N \log N)$ operations on a CREW PRAM, provided that the distance matrix of $Q_{n}$ is given. We also show that a graph on $N=2^{n}$ nodes can be recognized whether it is an $n$-dimensional hypercube in $\mathrm{O}(\log N)$ time using a total of $\mathrm{O}(N \log N)$ operations on a CRCW PRAM model or in $\mathrm{O}\left(\log ^{2} N\right)$ time using a total of $\mathrm{O}(N \log N)$ operations on a CREW PRAM model.

\section{References}

[1] D.P. Bertsekas, J.N. Tsitsiklis, Parallel and Distributed Computations: Numerical Computations, Prentice-Hall, Englewood Cliffs, NJ, 1989.

[2] D. Bhagavathi, C.E. Grosch, S. Olariu, A greedy hypercube-labeling algorithm, Comput. J. 37 (1994) 124-128.

[3] A. Coppesmith, S. Winogrand, Matrix multiplication via arithmetic progression in STOC'87, J. Symbolic Comput. 9 (1990) 251-280.

[4] T. Cormen, C. Leiserson, R. Rivest, Introduction to Algorithms, MIT Press/McGraw-Hill, Cambridge, MA/New York, 1991.

[5] S. Foldes, A characterization of hypercubes, Discrete Math. 17 (1977) 155-159.

[6] M.R. Garey, R.L. Graham, On cubical graphs, J. Combin. Theory B 16 (1975) 84-95.

[7] F. Harary, Graph Theory, Addison-Wesley, Reading, MA, 1969.

[8] F. Harary, J. Nieminen, Convexity in graphs, J. Differential Geometry 16 (1981) 185-190.

[9] J. JáJá, An Introduction to Parallel Algorithms, Addison-Wesley, Reading, MA, 1992.

[10] S.L. Johnsson, C.-T. Ho, Optimal broadcasting and personalized communications in hypercube, IEEE Trans. Comput. C 38 (1989) 1249-1268.

[11] V. Kumar, A. Grama, A. Gupta, G. Karypis, Introduction to Parallel Computing: Design and Analysis of Algorithms, Benjamin/Cummings, Menlo Park, CA, 1994.

[12] J.M. Laborde, S.P.R. Hebbare, Another characterization of hypercubes, Discrete Math. 39 (1982) 161-166.

[13] S. Lakshmivarahan, S.K. Dhall, Analysis and Design of Parallel Algorithms, McGraw-Hill, New York, 1990.

[14] S.D. Nikolopoulos, S.D. Danielopoulos, Parallel computation of perfect elimination schemes using partition techniques on triangulated graphs, Comput. Math. Appl. 29 (1995) 47-57. 
[15] J. Reif (Ed.), Synthesis of Parallel Algorithms, Morgan Kaufmann, San Mateo, CA, 1993.

[16] Y. Saad, M.H. Schultz, Topological properties of hypercube, IEEE Trans. Comput. C 37 (1988) 867-872.

[17] D.S. Scott, J. Brandenburg, Minimal mesh embedding in binary hypercubes, IEEE Trans. Comput. C 37 (1988) 1284-1288.

[18] A.Y. Wu, Embeddings of tree networks into hypercubes, J. Parallel Distributed Comput. 2 (1985) 238-249. 